\title{
Modality-specific spatial coordinates
}

\author{
N. O'CONNOR and B. HERMELIN \\ MRC Developmental Psychology Unit, Drayton House, Gordon Street, London, W. C.1, England
}

\begin{abstract}
The development of spatial coordinates may depend on the integration of information from different modalities, or may be based on modality-specific coding. On the first assumption, the blind would be handicapped in deriving adequate spatial concepts, and on the other, no such handicap need exist. When spatial orientation is the salient feature of the task, subjects appear to profit from the integration of information from two or more modalities. When shape is salient, subjects adopt modality-specific coding strategies.
\end{abstract}

In previous work, we (Hermelin \& O'Connor, 1971) slowed that a spatial task using tactile stimuli was solved in one way by blind and blindfolded and in another by sighted children. The presence or absence of visual cues atppeared to alter the coding process independently of prior visual experience. This would support al modality-specitic view of spatial constructs. It might suggest that the spatial reference system of blind as well as of sighted but blindfolded children differed from that of sighted children. One aspect of llis experiment was that the blindfolded, despite long risual experience, did not seem to transfer schemata derived from this experience to the task in hand.

Some investigators, like Held (1961) and Pribram (19-1). Believe that information is not necessarily corled only in terms of the sensory modality of input. Pribram (1971) bases this opinion on a suggestion made by sherrington (1947). He argues that the anatomical links between the sensory areas and the motor cortex might justify the view that the motor centers integrate the respective inputs of all receptors. Hhus. space perception would depend on the integration of all sensory imputs. However, Attneave and Benson (1969), Mckimney (1964), Warren (1970, and ron Senden $(1960)$ suggest that vision is the primary referent, and that normal conceptualization of space might require the mapping of tactile. hinacshletic or other sensory stimuli onto some referent schemal or image derived from visual experience. Thus, despite differences in emphasis, these theories hold that spatial perception depends on the cordination of data from different sense modialities.

for experimental purposes, the complexity of spatial experience needs to be further defined. Warren (Note 1) has suggested that it can be divided into spatial experience obtained through touch and therefore proximate to the body, or alternatively

We woulcl like to acknowledge the valuable comments and suggestions of Dr. U. Frith and Professor 1. P. Howard. and we would like to thank the headmaster. staft, and pupils of Dortonhouse Schuol and Islington Green School for their conperation. experience in extended space. Worchel (1951) made a lurther subdivision, i.e., the perception of form, the mental manipuation of form in space, and the orientation of the subject himself in space.

Our aim in the two experiments reported below is to determine the degree to which spatial concepts are dependent on one modality, such as vision, or the degree to which they are independent of any particulat modality of input. In the present context. ine are mainly concerned with the mental manipulation of forms in space, and with whether the information needed for such manipulation is or is not sense specilic. This has been approached experimentally in tho ways, by tigure composition (e.g.. Worchel. 1451) and by comparison or identification of ligures in different orientations (e.g., Cronin. 1972; Ghent. 1960). In the former, two separate shapes are individually explored by touch and the subject is required to describe or identify the form which would result from their juxtaposition. In the latter, the subject is required to recognize a known form in a variety of orientations. In both cases, the task is one of manipulating a mental image or images. Both kinds of lisk are known to be difficult, and the rotational tisk has been show'n to increase in difficulty according to the increasing angle of rotation in studies by shepard and Metzler (1971) and by Shinar and Owen (19-3). The latter consider that after practice an appreciation of rotation-independent features develops and substitutes for consideration of rotational liatures during comparison or recognition. However, as much of the work on rotation has been carried out visually by sighted children and some of that on figure composition has used touch with blind children, the comparative effects of input modality for the two kinds of operation have not been tested. Experiments "ith blind subjects obriously have a bearing on the releratuce of visual experience for facility in the matnipulation of forms in space. Worchel's (1951) results on the mental manipulation of tactually experienced spatial forms by the blind, derived from the comparison of congenitally blind, adventitiously blinded, and sighted subjects, showed that the sighted 
made many fewer errors than the blind and that the accidentally blinded were significantly superior to the congentically blind in the imaginal manipulation of space relations. Worchel noted that the use of visual imagery was a definite aid to the sighted and to the accidentally blinded in such manipulation. The figures used in the task employed by Worchel were. on the whole, well-known geometric shapes.

In the two experiments reported below. blind. sighted. and blindfolded subjects are compared in their performance on two tasks, one involving figure composition and the other figure orientation.

\section{EXPERIMENT I}

\section{Method}

Subjects. Blind and sighted children acted as subjects. Half the sighted were blindfolded. and there were 24 subjects in each of the three groups. The average age of each group was 13 years 7 months for the blind, 13 years 10 months for the blindfolded, and 13 years 8 months for the sighted. All children were of normal intelligence, as judged by scholastic estimates, and the Wechsler vocabularies of the three groups showed that verbal mental age did not differ from the mean chronological age in each group. All blind subjects had been blind from birth.

Material. The shapes were two-dimensional cutouts, based on forms used by Thurstone (1946) in his test of Primary Mental Abilities. A square with a missing piece was presented beside another piece, which in half the cases would fit into the gap in the square to complete it. In the other instances, the second piece would not fit into the gap in the square. These pairs of rwo-dimensional shapes were mounted alongside each other on a flat board measuring $30 \times 10 \mathrm{~cm}$. The larger piece was always mounted on the left-hand end of this backing board, and was itself $10 \mathrm{~cm}$ square when complete. In Figure 1, several examples of the shapes used are shown. Two sets of 20 pairs of shapes were prepared. In one set, half of the cut-out sections would fit directly into the gap in the square. In the other set, the smaller piece would first have to be rotated through $180^{\circ}$ before direct fitting was possible. The two pieces were fixed and could not be moved.

\section{Results}

The total number of errors for rotated and unrotated presentations for each group are set out in Table 1 . It was clear from the data that an overall analysis of variance would be inappropriate because of the lack of homogeneity of variance across the groups. For this reason, error scores were expressed as a proportion of the total possible score for each subject on rotated and unrotated shapes and converted to arc sine transformations. The analysis of variance was performed on these transformed scores. Though the main terms of groups and rotations were signiticant $(p<.001)$, there was also a Groups by

Table 1

Shapes: Total Error Scores by Groups and Presentations

\begin{tabular}{lccc}
\hline & Rotated & Unrotated & Total \\
\hline Blind & 139 & 124 & 263 \\
Blindfold & 154 & 159 & 313 \\
Sighted & 38 & 15 & 53 \\
Total & 331 & 298 & 629 \\
\hline
\end{tabular}

Note-Possible score per cell $=480$.
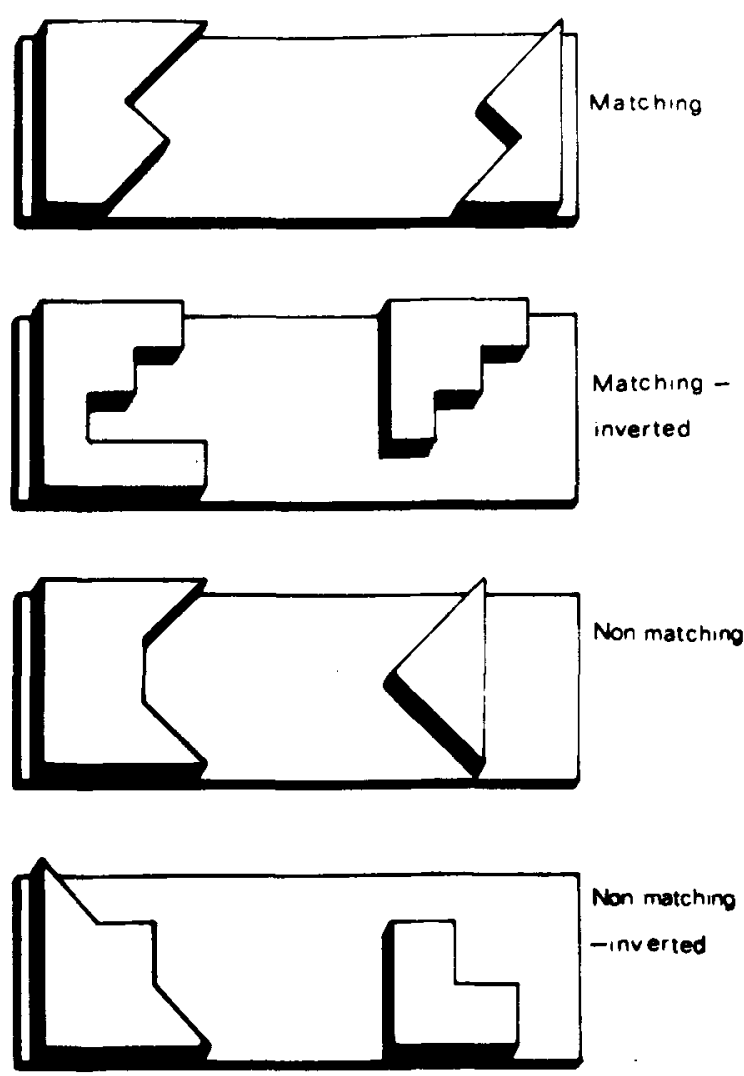

Figure 1. Examples of shapes used.

Rotations interaction $(\mathrm{F}=5.62$, $\mathrm{df}=2 / 138$, $\mathrm{p}<.01)$. Subsequent $t$ tests confirmed the impression of Table 1 that only the sighted group showed a signiticant difference between rotated and unrotated error scores $(t=4.56, p<.001)$. Nonparametric treatment of the basic scores confirmed this result.

It is also clear from Table 1 that, as expected, the "visual" group achieved better scores and fewer errors than the two tactile groups.

\section{Discussion}

One finding in this study was that, perhaps not surprisingly, figure completion was achieved more effectively by vision than by touch. More importantly. the effect of rotation found by other investigators with visually presented shapes was confirmed in this experiment, but in addition shown not to extend to tactile perception. It thus seems that the hand is less liable to take account of orientation as a crucial feature than the eye and that, as far as touch is concerned, rotation-independent features of the shapes were explored.

A possible reason for the apparent rotation independence of touch as compared with sight is that subjects may obtain their own self-referred "upright" orientation of an object by turning their hands through $180^{\circ}$ as some blind subjects did when 
exploring the shape for the identification of crucial features. It was almost as if the concept of spatial orientation independent of its relationship to the body had little significance for the blind.

Another finding of this experiment was that the previous visual experience of the blindfolded group which could have given rise to a visually based inference system was of no advantage in the mental manipulation of shapes by touch. Unlike the shapes in Worchel's (1951) study, those we used were not familiar, and, therefore, it could be the case that no ready visual images of them were formed by the blindfolded children on the basis of tactile exploration over only 40 trials. A comparison of the first 5 with the last 5 trials for each subject gave nearly identical error scores for the blind and blindfolded groups and showed an increase rather than a decrease in errors. Totals were 24 errors in each group for the first 5 trials and 41 for the blind and 46 for the blindfolded over the last 5 trials.

If the primacy of orientation were to be emphasized in a new task and that of shape reduced or minimized, an orientational schema based on visual experience might prove of greater advantage. Accordingly, the next experiment was designed to eliminate shape as far as possible as a discriminating feature and to cmphasize orientation in comparing the performance of blind, blindfolded, and sighted subjects.

\section{EXPERIMENT II}

\section{Method}

Subjects. As in the first experiment, the subjects were blind. blindfolded, and sighted child ren. They were 15 children from each of the previous groups, and though the groups were smaller. their coniprability on CA and MA was unaffected.

Material. Two plastic hands were moulded and prepared, a right hand and a left. The hands were moulded from casts of the hands of a colleague and were therefore realistic in size and proportion.

Procedure. The experimenter sat opposite the subject and presented him with a right or a left plastic hand in one of six orientations. and in four axial rotations for each of these orientations. The subject was required to say whether it was a left hand or a right hand. The six orientations were fingers pointing up vertically, down vertically, to the left, to the right, towards the experimenter, or away from him. The axial positions were in the four horizontal orientations of thumb up, thumb down, thumb left, or thumb right and in the two vertical orientations of thumb left, thumb right, thumb towards experimenter, or thumb away from him. Each of the resulting 24 test situations was presented twice, leading to 48 trials for each subject. Each subject went through a different randomization sequence. In the case of the sighted group. the subjects simply looked at the hand. The blind and blind folded children were asked to feel around the hand with their own preferred hands.

\section{Results}

The effect of axial rotations was considered first. Mean error scores for rotations are presented in Table 2. It can be seen that although total errors differ from group to group $(\mathrm{F}=11.62$. $\mathrm{df}=2 / 42$. $\mathrm{p}<.(0) 1)$, they do not differ between rotations $(\mathrm{F}=$ 1.01 , di $=3 / 126$, n.s.). Consequently, the four axial
Table 2

Mean Error Scores by Groups and Axial Rotations

\begin{tabular}{llcccc}
\hline & & \multicolumn{4}{c}{ Rotation } \\
\cline { 3 - 6 } & & 1 & 2 & 3 & 4 \\
\hline \multirow{2}{*}{ Blind } & Mean & 3.67 & 4.87 & 4.00 & 4.07 \\
& SD & 2.50 & 2.85 & 2.51 & 2.31 \\
Blindfold & Mean & 2.13 & 2.00 & 2.33 & 2.13 \\
& SD & 1.85 & 1.69 & 1.87 & 2.09 \\
Sighted & Mean & 1.00 & 1.13 & 1.67 & 1.47 \\
& SD & 1.19 & 1.68 & 1.54 & .92 \\
\hline
\end{tabular}

Table 3

Hands: Total Error Scores by Groups and Orientations

\begin{tabular}{lcccrrrr} 
& & & & \multicolumn{4}{c}{$\begin{array}{c}\text { To- } \\
\text { ward }\end{array}$} \\
& Up & Awom \\
& Down & Right & Left & \multicolumn{1}{c}{ E } & \multicolumn{1}{c}{ E } & Total \\
\hline Blind & 39 & 39 & 39 & 38 & 44 & 46 & 245 \\
Blindfold & 19 & 26 & 23 & 22 & 13 & 26 & 129 \\
Sighted & 10 & 15 & 16 & 7 & 9 & 22 & 79 \\
Total & 68 & 80 & 78 & 67 & 66 & 94 & 453 \\
\hline
\end{tabular}

Note-Possible score per cell $=120$.

rotation scores for each hand orientation were summated. The resulting basic error scores for hand orientations are presented in Table 3. As the variance of the visual group differed from the two tactile ones, these error scores were first expressed as proportions and then as arc sine transformations. An analysis of variance compared the three groups and the six orientations of the hand, i.e., away from and towards the experimenter, up and down, and left and right. There were two significant main effects, Groups ( $\mathrm{F}=$ 8.98, $\left.\mathrm{df}^{\circ}=2 / 42, \mathrm{p}<.001\right)$ and Orientations $(\mathrm{F}=$ 3.63, df $=5 / 210, p<.01)$. Subsequent $t$ tests showed that the blind made more errors than the blindfolded $(\mathrm{t}=6.6, \mathrm{p}<.001)$ and that the blindfolded made more than the sighted group $(t=$ 3.6, $p<.001)$. Comparison of orientations showed that the "away" and "towards" orientations differed, but that the "up" did not differ from the "down" orientation or the "right" from the "left." $t$ for the "away"."towards" difference was 4.1, p $<.001$. There was, however, no significant interaction between groups and orientations, despite the suggestion in the data of a difference between the "towards" and "away" positions for the blind and blindfolded but not for the sighted group.

In the search for the existence of such an interaction between groups and orientations, further analyses of variance, both parametric and nonparametric, were carried out using only the "towards" and "away" data and ignoring other error scores which may have obscured this possible interaction. None of these analyses showed a significant interaction between groups and positions. An analysis based on "away" minus "towards" difference scores also failed to show any difference between the groups. On the other hand, simple 
related t tests showed that scores for the blind did not differ for the two orientations, whereas scores for both the blind folded $(t=2.72, p<.02)$ and the sighted $(t$ $=2.43 . p<.02)$ did. However. because of lack of signiticance in the analyses of variance, the use of a related $t$ in these circumstances seems questionable.

\section{Discussion}

From the results of the first experiment. it was clear that the blind and the blindfolded performed at approximately the same level when deciding whether or not one shape would fit into another. There was thus no advantage of previous visual experience in aiding a shape discrimination carried out tactually. whether the shapes were presented simply or whether they were rotated. One possible reason for this might be that the shapes were not sufficiently simple to allow for ready encoding into a visual image on the basis of tactile exploration. It seems unlikely that these results were affected by the lack of tactile experience by the blindfolded group as compared with the blind. Error rates increased rather than decreased in the course of the 40 trials and were closely similar for both these groups.

However, in the second experiment, form was made almost irrelevant and was. moreover, very familiar. Only the spatial orientations of the hands helped the subject to discern which was a right and which a left hand. In these conditions, a frame of reference. including visual dimensions, seems to have aided the blindfolded in comparison with the congenitally blind children. If the blindfolded were able to evoke visual images suggested by their tactile experience in this task, then the visual schema so evoked would appear to have aided identification.

In the first experiment. only the sighted group experienced difficulty in deciding whether shapes would fit into each other in the rotated presentation condition. In this respect, the pattern of error scores differed significantly from that of the two tactile groups. In the second experiment, it appeared as if the sighted as well as the blindfolded children showed an effect of some orientations which was not evident in the results of the blind. One could argue that the hand position with the fingers pointing toward the experimenter might be regarded as the most "normal" orientation from the subject's point of view. while the "away from the experimenter" condition might be the most "unnatural." But, though the difference in error scores between the "toward" and "away" conditions seemed more marked and consistent for the sighted and blindfolded than for the blind group. a variety of statistical tests failed to establish this at an acceptable level of significance.

Spatial orientation was apparently more salient in the hand orientation than in the shape-fitting task. In the latter, as Shinar and Owen (1973) have suggested. rotation-independent features might have been substituted for orientational ones. Such orientation- independent features might be less dependent on a visually derived reference system and might be distinguishable by touch alone. Thus, although, as one might expect. the form conpletion task was better done with vision than without, rotating the shapes made it relatively more difficult for the visual group which took account of the orientational feature.

Where rotation-independent features, such as slape characteristics. cannot be used in the solution of a task, for instance when orientation is the most salient feature, mapping tactile information onto a visual referent aids solution. Thus, in the second task, when the salience of shape was minimized and that of orientation maximized, visual schemata were easily evoked and helped the blindfolded children to perform better than the blind. In this instance, the mechanism involved was not modality-specific coding. but the integration of the tactile input and a primary visual spatial reference system.

\section{REFERENCES}

Attneave, F., \& Benson, B. Spatial coding of tactual stimulation. Joumal of Experimental Psychology, 1969. 81. 216-222.

Cronin. V. Orientation and position effects of mirror-image reversal discrinination of triangles by young children. Perceptual and Motor Skills. 1972, 34, 707-711.

GHENT, L. Recognition by children of realistic figures presented in various orientations. Canadian Journal of Psychology. 1960. 14. 249-256.

HELD, R. Exposure history as a factor in maintaining stability of perception and coordination. Journal of Nervous and Mental Diseases, 1961, 132. 26-32.

Hermelin. B., \& O'Connor. N. Spatial coding in normal, autistic and blind children. Perceptual and Motor Skills, 1971, 33. $127-132$.

Mc Kinney. J. P. Hand schema in children. Psychonomic Science, 1964. 1. 99-100.

Pribram, K. H. Languages of the brain. New York: PrenticeHall, 1971.

Shepard, R. N. \& Metzler, J. Mental rotation of threedimensional objects. Science, 1971, 171, 701-703.

SHERRINGTON, C. S. The integrative action of the nervous system. Cambridge: Cambridge University Press, 1947.

SHINAR, D., \& OWEN, D. H. Effects of form rotation on the speed of classitication. The development of shape constancy. Perception \& Psychophysics, 1973. 14. 149-154.

Thurstone, L. L. Primary mental abilities. Chicago: Science Research Associates. 1946.

von SENDEN, M. Space and sight. London: Methuen, 1960.

WARREN, D. H. Intermodality interactions in spatial localization. Cognitive Psychology. 1970. 1. 114-133.

Worchel. P. Space perception and orientation in the blind. Psychological Monographs, 1951, 65, 1-28.

\section{NOTE}

1. Warren. D, H. Early vs. late blindness: The role of early vision in spatial reference systems. Paper presented at Meeting of Society for Research in Child Development. March 1973.

(Received for publication August 5, 1974: accepted November 8 . 1974.) 